\title{
Liver transplantation after DRESS syndrome: a case report and review of the literature
}

\author{
Igor Calil ${ }^{1}$, FRANCISCO TUSTUMI ${ }^{2}$, Rafael Pinheiro ${ }^{1}$, Ryan Tanigawa ${ }^{2}$, Jorge de Sousa ${ }^{1}$, \\ Ruy Cruz Junior ${ }^{2}$, Luiz D'Albuquerque ${ }^{2}$, and Rafael Pecora ${ }^{1}$ \\ ${ }^{1}$ Hospital Israelita Albert Einstein \\ ${ }^{2}$ Universidade de Sao Paulo Hospital das Clinicas
}

April 28, 2020

\begin{abstract}
Drug reaction with eosinophilia and systemic symptoms is a quite unusual condition related to drug reaction. A case report of sulfasalazine-induced liver failure is described. The patient was submitted to liver transplantation. Liver transplantation is an option when DRESS is associated with acute liver failure, but the prognosis remains poor.
\end{abstract}

\section{KEY CLINICAL MESSAGE}

This study describes a patient with drug reaction with eosinophilia and systemic symptoms (DRESS syndrome), associated with liver failure.

\section{INTRODUCTION}

Drug reaction with eosinophilia and systemic symptoms (DRESS syndrome) is a quite unusual condition related to drug reaction. ${ }^{1}$ Several drugs have been linked to DRESS. It is a severe idiosyncratic drug reaction characterised by erythematous or papulo-pustular skin eruption associated with lymphadenopathy, fever, and visceral involvement (hepatitis, nephritis pneumonitis, pericarditis, myocarditis, and colitis) ${ }^{2-6}$ Leucocytosis, eosinophilia (90\%) and/or mononucleosis (40\%) also may be seen. ${ }^{6}$ Severe acute hepatitis due to sulfasalazine or Trimethoprim-sulfamethoxazole is described in literature, but the occurrence of DRESS and liver failure is rare.

In this study, we report a patient with acute liver failure due to sulfasalazine-induced DRESS, treated with liver transplant.

\section{CASE REPORT}

An 18-year-old male patient was treated for toxoplasma retinochoroiditis with sulfasalazine, along one month. The patient had no past history of allergies or drug intolerance. He presented in a local hospital with fever, vomiting, cervical and inguinal nodules, abdominal pain, and macular rash on all body. The patient was transferred to our Transplant Center after onset of jaundice and encephalopathy. He was admited in intensive care unit with facial edema, generalized scaling exanthema and acute hepatitis. Serological tests for viral hepatitis and all autoimmune antibodies were negative. Laboratory tests showed a total eosinophil count of $3220 / \mathrm{mm}^{3}$ (normal, $<500 \mathrm{~mm}^{3}$ ), high level of transaminases (AST=1303 IU/L; ALT=1768 IU/L, lactate dehydrogenase level of $2274 \mathrm{IU} / \mathrm{L}$ (normal, 240 to $480 \mathrm{IU} / \mathrm{L}$ ), total bilirubin level of $18.47 \mathrm{mg} / \mathrm{dL}$, direct bilirubin level of $14.81 \mathrm{mg} / \mathrm{dL}$, prothrombin time (PT) international normalized ratio (INR) of 5.18, and Factor V 17\%. Abddominal ultrasound examination identified no cronical liver disease. The RegiSCAR ${ }^{7}$ 
system scored 5 points confirming the diagnosis of DRESS. Skin biopsy observed Interface and spongiotic dermatitis, consistent with drug eruption.

Therefore, the patient was worked up for urgent orthotopic liver transplantation (OLT), which was performed 24 hours after admission. At this time he was under corticosteroids and clinical support, including mechanic ventilation due progressive encephalopathy and dyalisis due latic acydosis.

The orthotopic liver transplantation was uneventfull. Even though liver function improved in postoperative period, the patient developed sepsis requiring high doses of vasopressors. Broad-spectrum antibiotics were introduced but patient remained hemodynamic unstable. The paitient died at $7^{\text {th }}$ postoperative day. Blood cultures showed growth of aKlebsiella pneumoniae resistent to carbapenems.

\section{Liver and Skin histology}

The histology analisis demonstred massive eosinophils infintrat compatible with DRESS and the liver explant presented a massive necrose associated a eosinophilis infiltrated (Figure 1 and 2).

\section{DISCUSSION}

The present report describes an adult patient with DRESS syndrome and liver failure treated with OLT. Liver failure in the setting of DRESS syndrome is quite rare. Few case reports presented patients with DRESS syndrome and high level of hepatic injury (Table 1). In these studies, most of the patients were treated with corticosteroids ${ }^{8-29}$.

The management of DRESS syndrome is challenging. It is important to withdraw the suspected drug and the delay is associated with poorer outcomes. ${ }^{30-32}$ Supportive therapy in intensive care unit should be provided to stabilize the patient. Early administration of systemic corticosteroid therapy is generally recommended. ${ }^{33}$ Systemic corticosteroid helps to improve in both clinical symptoms and laboratory abnormalities within days. ${ }^{33}$ Most of the cases reports of DRESS syndrome with liver dysfunction showed success with corticosteroids treatment (Table 1 ).

Liver transplantation is an option when DRESS is associated with acute fulminant hepatic failure, but the prognosis remains poor (Table 2) ) $^{34-39}$. Besnard et al. ${ }^{34}$ reported two pediatric Crohn's disease patients undergoing liver transplantation after DRESS syndrome induced by sulfasalazine. During follow-up, one of them developed acute rejection and fatal aspergilosis. Song et al. ${ }^{37}$ reported living-donor liver transplantation in a 14-years old patient. Patient presented chronic rejection after 25 -months follow-up. Amante et al. ${ }^{35}$ and Roales-Gómez et al. ${ }^{38}$ reported adult patients treated with OLT, with no information concerning long-term follow-up. Mennickea et al. ${ }^{36}$ reported an adult patient treated with OLT, with mortality in postoperative period due to massive intrabominal blood loss.

Recent studies support the use of Molecular Adsorbents Recirculation System (MARS) as a rescue for patients with liver failure. Roales-Gómez et al. ${ }^{38}$ described MARS use, although patient did not responde well, and patients was eventually submmited to OLT. $\mathrm{Ng}$ et al. ${ }^{23}$ reported a pediatric patient that underwent MARS in the intensive care unit, with satisfactory response.

The present study showed a patient with Sulfasalazine and Trimethoprim-sulfamethoxazole severe reaction. Sulfasalazine and Trimethoprim-sulfamethoxazole carries a significant risk of drug toxicity. Yusuf et al. ${ }^{24}$ reported the first case of DRESS syndrome in a child treated for toxoplasma retinochoroiditis. Rare cases of immunoallergic reactions to sulfasalazine, including DRESS syndromes, have been reported, such as the classic " 3 -week sulfasalazine syndrome" occurring 3 weeks after the first administration. ${ }^{8}$

\section{CONCLUSION}

DRESS is associated with acute liver failure is a lifethreatening condition. Liver transplantation is an option for the management of these patients, although the prognosis remains poor.

\section{AUTHORS CONTRIBUTIONS:}


- Igor Lepski Calil ${ }^{3}, \mathrm{PhD}$ : (analysis and interpretation of data)

- Rafael Soares Nunes Pinheiro ${ }^{1}, \mathrm{PhD}$ : (acquisition of data and drafting the article)

- Ryan Yukimatsu Tanigawa ${ }^{2}$, MD: (paper drafting)

- Francisco Tustumi ${ }^{1}, \mathrm{PhD}$ : (paper drafting)

- Rafael Antônio Arruda Pecora ${ }^{3}, \mathrm{PhD}$ : (revising the paper critically for relevant intellectual content)

- Ruy Jorge Cruz Junior ${ }^{1}$, MD: (revising the paper critically for valuable intellectual content)

- Luiz Augusto Carneiro D'Albuquerque ${ }^{1}, \mathrm{PhD}$ : (conception and design of the study)

- Orlando de Castro e Silva Júnior ${ }^{1} \mathrm{PhD}$ : (final approval of the version to be submitted)

\section{REFERENCES}

1. Newell BD, Moinfar M, Mancini AJ, Nopper AJ. Retrospective analysis of 32 pediatric patients with anticonvulsant hypersensitivity syndrome (ACHSS). Pediatr Dermatol 2009;26:536-46.

2. Bocquet H, Bagot M, Roujeau JC. Drug-induced pseudolymphoma and drug hypersensitivity syndrome (drug rash with eosinophilia and systemic symptoms: DRESS). Semin Cutan Med Surg 1996;15:250-7.

3. Naisbitt DJ, Britschgi M, Wong G et al. Hypersensitivity reactions to carbamazepine: characterisation of the specificity, phenotype and cytokine profile of drug-specific T-cell clones. Mol Pharmacol2003; 63: $732-41$.

4. Naisbitt DJ, Farrell J, Wong G et al. Characterisation of drugspecific T cells in lamotrigine hypersensitivity. J Allergy Clin Immunol 2003 ; 111: 1393-403.

5. Eland IA, Dofferhoff AS, Vink R, Zondervan PE, Stricker BH. Colitis may be part of the antiepileptic drug hypersensitivity syndrome. Epilepsia 1999; 40: 1780-83.

6. Callot V, Roujeau JC, Bagot M et al. Drug induced pseudolymphoma and hypersensitivity syndrome. Two different clinical entities. Arch Dermatol 1996; 132: 1315-21.

7. Kardaun SH, Sidoroff A, Valeyrie-Allanore L, et al. Variability in the clinical pattern of cutaneous side-effects of drugs with systemic symptoms: does a DRESS syndrome really exist? $\mathrm{Br} J$ Dermatol . 2007;156:609-611.

8. Brooks H, Taylor HG, Nichol FE (1992) The three week sulphasalazine syndrome. Clin Rheumatol 11:566-568

9. Queyrel V, Catteau B, Michon-Pasturel U, Fauchais AL, Delcey V, Launay D, Legout L, Hachulla E, Hatron PY, Devulder B.DRESS (Drug Rash with Eosinophilia and Systemic Symptoms) syndrome after sulfasalazine and carmazepine: report of two cases.Rev Med Interne. 2001 Jun;22(6):582-6.

10. Mainra,RR, Card,SE.Trimethoprim-sulfamethoxazole-associated hepatotoxicity - part of a hypersensitivity syndrome. Can J Clin Pharmacol. 2003 Winter;10(4):175-8.

11. Descloux E, Argaud L, Dumortier J, Scoazec JY, Boillot O, Robert D. Favourable issue of a fulminant hepatitis associated with sulfasalazine DRESS syndrome without liver transplantation. Intensive Care Med. 2005 Dec;31(12):1727-8

12. Michel,F, Navellou,JC, Ferraud,D, Toussirot,E, Wendling,D.DRESS syndrome in a patient on sulfasalazine for rheumatoid arthritis. Joint Bone Spine. 2005 Jan;72(1):82-5.

13. Teo L, Tan E. Sulphasalazine-induced DRESS. Singapore Med J 2006; 47(3):237-239

14. Bejia I, Ben Hammouda S, Riahi K, Zinelabidine F, Mediouni B, Touzi M, Bergaoui N. DRESS syndrome induced by sulphasalazine in rheumatoid arthritis. Joint Bone Spine . 2006 Dec;73(6):764-5.

15. Aquino RT, Vergueiro CS, Magliari ME, de Freitas TH. Sulfasalazine-induced DRESS syndrome (Drug Rash with Eosinophilia and Systemic Symptoms).Sao Paulo Med J. 2008 Jul;126(4):225-6.

16. Augusto JF, Sayegh J, Simon A, Croue A, Chennebault JM, Cousin M, Subra JF. A case of sulphasalazineinduced DRESS syndrome with delayed acute interstitial nephritis. Nephrol Dial Transplant. 2009 Sep;24(9):29402 .

17. Yeşilova,Z, Kantarcioğlu,M, Erçin,CN, Safalioğlu,M, Kilciler,G, Koç,E, Atli,M, Uygun,ASulfasalazineinduced hypersensitivity: a case report of DRESS syndrome. Turk J Gastroenterol. 2009 Dec;20(4):298-9

18. Rosenbaum J, Alex G, Roberts H, Orchard D. Drug rash with eosinophilia and systemic symptoms secondary to sulfasalazine.J Paediatr Child Health . 2010 Apr;46(4):193-6.

19. van der Mark SC ${ }^{1}$, Segers D, Bakker RC, van Wijngaarden, P.DRESS syndrome as a result of sulfasa- 
lazine use. Ned Tijdschr Geneeskd. 2010;154(18):A2161

20. Piñana E, Lei SH, Merino R, Melgosa M, De La Vega R, Gonzales-Obeso E, Ramírez E, Borobia A, Carcas A. DRESS-syndrome on sulfasalazine and naproxen treatment for juvenile idiopathic arthritis and reactivation of human herpevirus 6 in an 11-year-old Caucasian boy.J Clin Pharm Ther. 2010 Jun;35(3):365-70

21. Lau G, Kwan C, Meng Chong S (2001) The 3-week sulphasalazine syndrome strikes again. Forensic Sci Int 122:79-84.

22. Daoulah A, Alqahtani AA, Ocheltree SR, Alhabib A, Ocheltree AR. Acute myocardial infarction in a 56-year-old female patient treated with sulfasalazine.Am. J Emerg Med. 2012 May;30(4):638.e1-3.

23. Ng CT, Tan CK, Oh CC, Chang JP. Successful extracorporeal liver dialysis for the treatment of trimethoprim-sulfamethoxazole-induced fulminant hepatic failure. Singapore Med J. 2013 May 1;54(5):e1136.

24. Yusuf IH, Sahare P, Hildebrand GD. DRESS Syndrome in a child treated toxoplasma retinochoroiditis. $J$ AAPOS. 2013 Oct;17(5):521-3

25. Girelli,F, Bernardi,S, Gardelli,L, Bassi,B, Parente,G, Dubini,A, Serra,L, Nizzoli,MA New Case of DRESS Syndrome Induced by Sulfasalazine and Triggered by Amoxicillin.Case Rep Rheumatol. 2013;2013:409152.

26. Hernández,N, Borrego,L, Soler,E, Hernández,J. Sulfasalazine-induced linear immunoglobulin A bullous dermatosis with DRESS.Actas Dermosifiliogr. 2013 May;104(4):343-6.

27. Zaïem A, Aouinti I, Lakhoua G, Sahnoun R, Daghfous R, Lakhal M, El Aïdli S. Sulfasalazine-induced DRESS syndrome associated with Epstein Barr virus reactivation. Therapie . 2013 Sep-Oct;68(5):321-3.

28. Ferrero, NA, Pearson KC, Zedek DC, Morrell DS. Case report of drug rash with eosinophilia and systemic symptoms demonstrating human herpesvirus-6 reactivation.Pediatr Dermatol. 2013 SepOct;30(5):608-13.

29. Pirklbauer,M, Gruber,J. DRESS syndrome following sulfasalazine treatment.Z. Rheumatol. 2014 Mar;73(2):1803 .

30. Bachot N, Roujeau JC. Differential diagnosis of severe cutaneous drug eruptions. Am J Clin Dermatol 2003;4:561-72.

31. Tas S, Simonart T. Drug rash with eosinophilia and systemic symptoms (DRESS syndrome). Acta Clinica Belgica 1999;54: 197-200.

32. Tas S, Simonart T. Management of drug rash with eosinophilia and systemic symptoms (DRESS syndrome): an update. Dermatology2003;206:353-6.

33. Chiou CC, Yang LC, Hung SI, Chang YC, Kuo TT, Ho HC, et al. Clinicopathological features and prognosis of drug rash with eosinophilia and systemic symptoms: a study of 30 cases in Taiwan. $J$ Eur Acad Dermatol Venereol 2008;22: 1044-9.

34. Besnard M, Debray D, Durand P, Cezard JP, Navarro J (1998) Sulfasalazine-induced fulminant hepatitis in pediatric Crohn's disease: report of two cases. J Pedriatr Gastroenterol Nutr26:119-120.

35. Amante MF, Filippini AV, Cejas N, Lendoire J, Imventarza O, Parisi C. Dress syndrome and fulminant hepatic failure induced by lamotrigine. Ann Hepatol. 2009 Jan-Mar;8(1):75-7.

36. Mennicke M, Zawodniak A, Keller M, Wilkens L, Yawalkar N, Stickel F, Keogh A, Inderbitzin D, Candinas D, Pichler WJ. Fulminant liver failure after vancomycin in a sulfasalazine-induced DRESS syndrome: fatal recurrence after liver transplantation. American journal of transplantation. 2009 Sep;9(9):2197-202.

37. Song SM, Cho MS, Oh SH, Kim KM, Park YS, Kim DY, Lee SG. Liver transplantation in a child with acute liver failure resulting from drug rash with eosinophilia and systemic symptoms syndrome. Korean J Pediatr 2012;56(5):224-226

38. Roales-Gomez V, Molero AI, Perez-Amarilla I, Casabona-Frances S, Rey-Diaz-Rubio E, Catalan M, Vanaclocha F, Colina F DRESS syndrome secondary to ibuprofen as a cause of hyperacute liver failure.Rev Esp Enferm Dig. 2014 Aug;106(7):482-6.

\begin{tabular}{lllll}
\hline Case Report & Pacient & Drug & Treatment & Follow up \\
\hline Brooks H et al ${ }^{16}$ & 53-year-old man & Sulfasalazine & corticosteroids & Alive
\end{tabular}




\begin{tabular}{|c|c|c|c|c|}
\hline Case Report & Pacient & Drug & Treatment & Follow up \\
\hline Queyrel $\mathrm{V}$ et $\mathrm{al}^{35}$ & $\begin{array}{l}\text { 15-year-old } \\
\text { woman }\end{array}$ & Sulphasalazine & corticosteroids & Alive \\
\hline Mainra RR et $\mathrm{al}^{36}$ & $\begin{array}{l}\text { 24-year-old } \\
\text { woman }\end{array}$ & $\begin{array}{l}\text { Trimethoprim- } \\
\text { sulfamethoxazole }\end{array}$ & corticosteroids & Alive \\
\hline Descloux E et $\mathrm{al}^{37}$ & $\begin{array}{l}\text { 45-year-old } \\
\text { woman }\end{array}$ & Sulphasalazine & corticosteroids & Alive \\
\hline Michel F et al. ${ }^{38}$ & $\begin{array}{l}\text { 63-year-old } \\
\text { woman }\end{array}$ & sulfasalazine & corticosteroids & Alive \\
\hline Teo L et $\mathrm{al}^{14}$ & $\begin{array}{l}\text { 49-year-old } \\
\text { woman }\end{array}$ & Sulphasalazine & corticosteroids & Alive \\
\hline Bejia I et al ${ }^{39}$ & $\begin{array}{l}46 \text {-year-old } \\
\text { woman }\end{array}$ & Sulphasalazine & corticosteroids & Alive \\
\hline $\begin{array}{l}\text { de Aquino RT et } \\
\mathrm{al}^{40}\end{array}$ & $\begin{array}{l}47 \text {-year-old } \\
\text { woman }\end{array}$ & Sulphasalazine & corticosteroids & Alive \\
\hline $\begin{array}{l}\text { Augusto JF et } \\
\mathrm{al}^{41}\end{array}$ & $\begin{array}{l}\text { 77-year-old } \\
\text { woman }\end{array}$ & Sulphasalazine & corticosteroids & Alive \\
\hline Yeşilova $\mathrm{Z}$ et al ${ }^{43}$ & 38-year-old man & Sulphasalazine & corticosteroids & Alive \\
\hline $\begin{array}{l}\text { Rosenbaum, J et } \\
\text { al. }{ }^{44}\end{array}$ & $\begin{array}{l}\text { 11-year-old } \\
\text { woman }\end{array}$ & Sulphasalazine & corticosteroids & Alive \\
\hline $\begin{array}{l}\text { van der Mark SC } \\
\text { et al }{ }^{45}\end{array}$ & $\begin{array}{l}\text { 24-year-old } \\
\text { woman }\end{array}$ & Sulphasalazine & corticosteroids & Alive \\
\hline Piñana $\mathrm{E}$ et $\mathrm{al}^{46}$ & 11-year-old boy & $\begin{array}{l}\text { Sulphasalazine } \\
\text { /Naproxen }\end{array}$ & corticosteroids & Alive \\
\hline Lau G et al ${ }^{17}$ & $\begin{array}{l}\text { 34-year-old } \\
\text { woman }\end{array}$ & Sulphasalazine & corticosteroids & Died \\
\hline Daoulah A et al ${ }^{47}$ & $\begin{array}{l}\text { 56-year-old } \\
\text { woman }\end{array}$ & Sulphasalazine & corticosteroids & Died \\
\hline $\mathrm{Ng} \mathrm{CT}$ et $\mathrm{al}^{48}$ & 17 year-old male & $\begin{array}{l}\text { Trimethoprim- } \\
\text { sulfamethoxazole }\end{array}$ & MARS & Alive \\
\hline Yusuf IH et al ${ }^{15}$ & 15-year-old girl & Sulphasalazine & corticosteroids & Alive \\
\hline Girelli F et al ${ }^{49}$ & $\begin{array}{l}\text { 53-year-old } \\
\text { woman }\end{array}$ & $\begin{array}{l}\text { Sulfasalazine/ } \\
\text { Amoxicillin }\end{array}$ & corticosteroids & Alive \\
\hline $\begin{array}{l}\text { Hernández } \mathrm{N} \text { et } \\
\mathrm{al}^{50}\end{array}$ & $\begin{array}{l}60 \text {-year-old } \\
\text { woman }\end{array}$ & Sulphasalazine & corticosteroids & Alive \\
\hline Zaïem A et $\mathrm{al}^{51}$ & $\begin{array}{l}45 \text {-year-old } \\
\text { woman }\end{array}$ & Sulphasalazine & corticosteroids & Alive \\
\hline Ferrero NA et al ${ }^{52}$ & 15-year-old boy & Sulphasalazine & corticosteroids & Alive \\
\hline $\begin{array}{l}\text { Pirklbauer M et } \\
\mathrm{al}^{53}\end{array}$ & $\begin{array}{l}\text { A 53-year-old } \\
\text { woman }\end{array}$ & Sulphasalazine & corticosteroids & Alive \\
\hline
\end{tabular}

Table 1. Reported cases who had diagnosis of DRESS caused by associated Sulfasalazine and Trimethoprimsulfamethoxazole

\begin{tabular}{llll}
\hline Case Report & Pacient & Drug & Follow up \\
\hline Besnard $\mathrm{M}$ et al ${ }^{18}$ & 10-year-old male & Sulfasalazine & Died \\
Besnard M et al ${ }^{18}$ & 10-year-old female & Sulfasalazine & Alive \\
Amante MF et al & 21-year old female & lamotrigine & Unknown \\
Mennickea M et al & 60-year-old male & Sulfasalazine/ & Died \\
& & vancomycin &
\end{tabular}




\begin{tabular}{llll}
\hline Case Report & Pacient & Drug & Follow up \\
\hline Song S et al ${ }^{55}$ & 14-year-old female & Vancomycin & Alive \\
Roales-Gómez V et al & 22-year-old male & Ibuprofen & Alive \\
P6 & 18-year-old male & Sulfamethoxazole & Died \\
\hline
\end{tabular}

Table 2. Patient diagnosed with DRESS undergoing liver transplantation.

\section{Caption}

Figure 1. Liver Histology. H\&E stain. Massive eosinophils infintrat with extensive necrosis of the liver compatible with fulminant hepatites.

Figure 2. Skin Histology. H\&E stain. Massive eosinophils infintrat compatible with DRESS

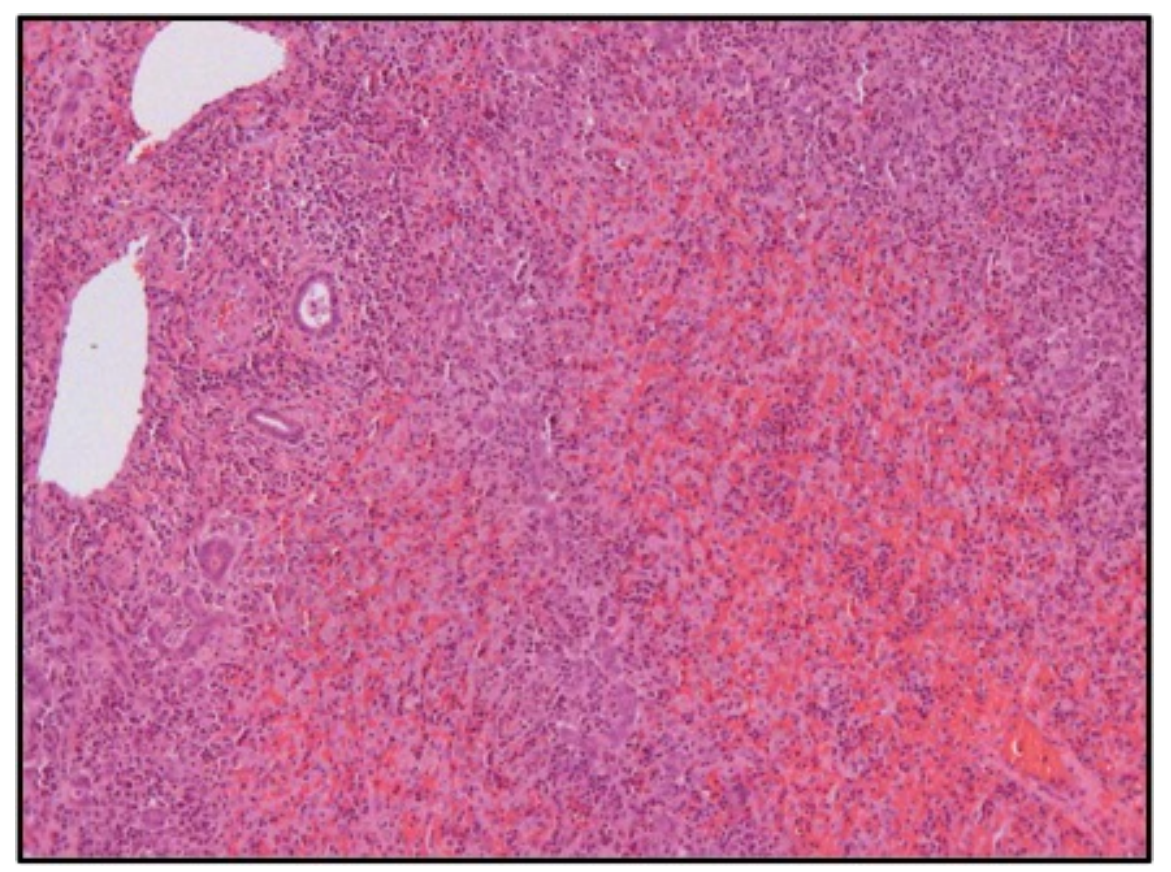




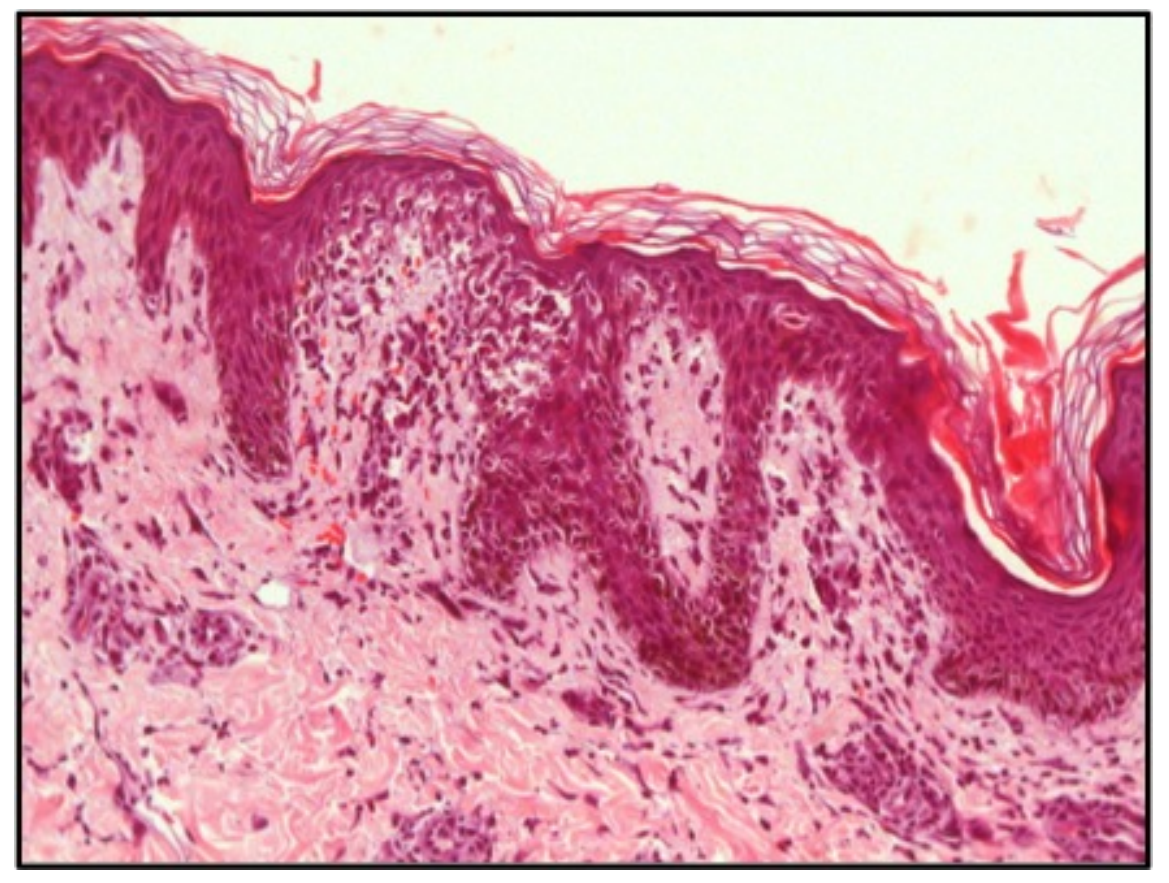

